\title{
Weierstrass type approximation by weighted polynomials in $\mathbb{R}^{d *}$
}

\author{
András Kroó ${ }^{\dagger}$
}

June 7, 2019

\begin{abstract}
In this paper we consider weighted polynomial approximation on unbounded multidimensional domains in the spirit of the weighted version of the Weierstrass trigonometric theorem according to which every continuous function on the real line with equal finite limits at $\pm \infty$ is a uniform limit on $\mathbb{R}$ of weighted algebraic polynomials of degree $2 n$ with varying weights $\left(1+t^{2}\right)^{-n}$. We will verify a similar statement in the multivariate setting for a general class of convex weights.

We also consider the similar problem of multivariate polynomial approximation with varying weights for some typical non convex weights. In case of non convex weights of the form $w_{\alpha}(\mathbf{x}):=\left(1+\left|x_{1}\right|^{\alpha}+\ldots+\left|x_{d}\right|^{\alpha}\right)^{\frac{1}{\alpha}}, 0<\alpha<1$ in order for weighted polynomial approximation to hold for a given continuous function it is necessary that the function vanishes on a certain exceptional set consisting of all coordinate hyperplanes and $\infty$. Moreover, in case of rational $\alpha$ this condition is also sufficient for weighted polynomial approximation to hold.
\end{abstract}

\section{Introduction}

Let us start by recalling the trigonometric version of the classical Weierstrass approximation theorem which states that any $2 \pi$ periodic continuous function $f(x)$ is a uniform limit on $[-\pi, \pi]$ of trigonometric polynomials of degree $n$ as $n \rightarrow \infty$. Clearly the substitution $t=\tan \frac{x}{2}, x \in(-\pi, \pi)$ transforms $2 \pi$ periodic continuous functions into continuous functions on $\mathbb{R}$ which have equal finite limits at $\pm \infty$, while the trigonometric polynomials of degree $n$ transform into rational functions $\left(1+t^{2}\right)^{-n} p_{2 n}(t)$ with $p_{2 n}(t)$ being an algebraic polynomial of degree at most $2 n$.

This results in the following equivalent version of the trigonometric Weierstrass theorem:

Every $f \in C(\mathbb{R})$ with equal finite limits at $\pm \infty$ is a uniform limit on $\mathbb{R}$ of weighted algebraic polynomials

$$
w(t)^{-2 n} p_{2 n}(t), \quad w(t):=\sqrt{1+t^{2}}, \operatorname{deg} p_{2 n} \leq 2 n .
$$

Naturally this leads to the question of uniform approximation by general weighted polynomials of the form

$$
w(t)^{-n} p_{n}(t), \operatorname{deg} p_{n} \leq n
$$

This question received a considerable attention in the recent years in case when the even weight $w(t)$ grows at $\infty$ faster than $t$, i.e., $\frac{w(t)}{t} \rightarrow \infty$ as $t \rightarrow \infty$, see for instance monographs [5] and [6]. The assumption $\frac{w(t)}{t} \rightarrow \infty$ obviously implies that $w(t)^{-n} p_{n}(t) \rightarrow 0, t \rightarrow \pm \infty$ for all polynomials of degree at most $n$. Thus, naturally under condition $\frac{w(t)}{t} \rightarrow \infty, t \rightarrow \infty$ weighted polynomials can not provide uniform approximation on all of the real line. Indeed, various results given in [5] and [6] show that only continuous functions with finite support can be approximated by weighted polynomials in this case, and in fact this finite domain of approximation which depends on $w$ can be determined by methods of potential theory.

In this paper we intend to consider weighted approximation on unbounded domains in the spirit of the weighted version of Weierstrass trigonometric approximation theorem. As mentioned above if $\frac{w(t)}{t}$ tends to infinity as $t \rightarrow \infty$ only functions with finite support can be approximated, i.e. approximation on the whole real line is not possible in this case. On the other hand if $\frac{w(t)}{t} \rightarrow 0$ as $t \rightarrow \infty$ then weighted polynomials $w(t)^{-n} p_{n}(t)$ are unbounded as $t \rightarrow \infty$

*AMS Subject classification: 41A10, 41A63. Key words and phrases: approximation by multivariate weighted polynomials, homogeneous polynomials, varying weights, convex weights, density

†Supported by the NKFIH - OTKA Grant K128922 
which also makes the approximation on unbounded domains impossible. Therefore we will require that the weights have linear growth at infinity. Moreover, this problem will be investigated below in the multivariate setting.

Let us denote by $S^{d-1}$ the Euclidian unit sphere in $\mathbb{R}^{d}$ and consider the space $C_{0}\left(\mathbb{R}^{d}\right)$ of continuous functions on $\mathbb{R}^{d}$ which have equal continuous limits at infinity along lines passing through the origin, i.e.,

$$
C_{0}\left(\mathbb{R}^{d}\right):=\left\{f \in C\left(\mathbb{R}^{d}\right): \exists r_{f} \in C\left(S^{d-1}\right) \text { such that } \lim _{|t| \rightarrow \infty} f(t \mathbf{x})=r_{f}(\mathbf{x}), \text { uniformly for } \mathbf{x} \in S^{d-1}\right\} .
$$

Note that for every $f \in C_{0}\left(\mathbb{R}^{d}\right)$ and $\mathbf{x} \in \mathbb{R}^{d} \backslash\{0\}$ we have that

$$
\lim _{t \rightarrow \pm \infty} f(t \mathbf{x})=r_{f}\left(\frac{\mathbf{x}}{|\mathbf{x}|}\right)
$$

and therefore it is natural to extend $r_{f} \in C\left(S^{d-1}\right)$ to $\mathbb{R}^{d} \backslash\{0\}$ by relation

$$
r_{f}(\mathbf{x}):=r_{f}\left(\frac{\mathbf{x}}{|\mathbf{x}|}\right), \mathbf{x} \in \mathbb{R}^{d} \backslash\{0\} .
$$

Let $w \in C\left(\mathbb{R}^{d}\right), w(-\mathbf{x})=w(\mathbf{x}), \forall \mathbf{x} \in \mathbb{R}^{d}$ be a positive even weight function on $\mathbb{R}^{d}$. We will consider uniform approximation of functions $f \in C_{0}\left(\mathbb{R}^{d}\right)$ by weighted polynomials $w^{-n} p_{n}$ on $\mathbb{R}^{d}$, where $p_{n} \in P_{n}^{d}$ are multivariate polynomials of $d$ variables of degree at most $n$. We will assume throughout this paper that $w$ is a positive continuous even weight on $\mathbb{R}^{d}$ such that $t w\left(\frac{\mathbf{x}}{t}\right)$ is monotone increasing for $t>0$ for every fixed $\mathbf{x} \in \mathbb{R}^{d}$, and has a continuous positive limit as $t \rightarrow 0$. This means that with some even positive continuous function

$$
\tilde{w}: S^{d-1} \mapsto \mathbb{R}^{+}
$$

we have by the Dini theorem that

$$
|t| w\left(\frac{\mathbf{x}}{t}\right) \rightarrow \tilde{w}(\mathbf{x}), \quad t \rightarrow 0, \quad \text { uniformly for } \mathbf{x} \in S^{d-1} .
$$

Hence in particular, $w(t \mathbf{x}) \sim|t| w(\mathbf{x}), t \rightarrow \infty$, i.e. the weight is of order $|t|$ at infinity. Evidently, extending $\tilde{w} \in C\left(S^{d-1}\right)$ to $\mathbb{R}^{d} \backslash\{0\}$ according to the relation $\tilde{w}(\alpha \mathbf{x})=|\alpha| \tilde{w}(\mathbf{x}), \alpha \in \mathbb{R}$ preserves (1) for every $\mathbf{x} \in \mathbb{R}^{d}$. In particular, when $d=1$ we have that $\tilde{w}(t) \equiv c|t|, t \in \mathbb{R}$ with some $c>0$.

In what follows positive continuous even weights $w$ on $\mathbb{R}^{d}$ for which $t w\left(\frac{\mathbf{x}}{t}\right)$ is monotone increasing with respect to $t>0$ for every fixed $\mathbf{x} \in \mathbb{R}^{d}$ and (1) is satisfied with certain positive continuous function $\tilde{w}$ will be called admissible. It is important to observe that for admissible weights $w$ we have $w^{-2 n} p_{2 n} \in C_{0}\left(\mathbb{R}^{d}\right)$ for any $p_{2 n} \in P_{2 n}^{d}$ and $n \in \mathbb{N}$.

For an admissible weight $w$ we consider its homogenization defined for every $(\mathbf{x}, t) \in \mathbb{R}^{d+1} \backslash\{0\}$ by

$$
w^{*}(\mathbf{x}, t):=\left\{\begin{array}{l}
|t| w\left(\frac{\mathbf{x}}{t}\right), \mathbf{x} \in \mathbb{R}^{d}, t \in \mathbb{R} \backslash\{0\} \\
\tilde{w}(\mathbf{x}), t=0 .
\end{array}\right.
$$

It is easy to see that this function $w^{*}: \mathbb{R}^{d+1} \mapsto \mathbb{R}$ possesses the homogeneous property

$$
w^{*}(c \mathbf{x}, c t)=|c| w^{*}(\mathbf{x}, t), \forall c \in \mathbb{R},(\mathbf{x}, t) \in \mathbb{R}^{d+1} .
$$

In addition, $w^{*}(\mathbf{x}, t)$ is positive everywhere in $\mathbb{R}^{d+1} \backslash\{0\}$ and we may set preserving continuity $w^{*}(0)=0$. Evidently, it follows from (1) that the above relation defines a continuous weight $w^{*}(\mathbf{x}, t) \in C\left(\mathbb{R}^{d+1}\right)$ since both $w$ and $\tilde{w}$ are continuous. Also note that $w^{*}(\mathbf{x}, t)$ is even both in $\mathbf{x} \in \mathbb{R}^{d}$ and $t \in \mathbb{R}$.

The problem of approximation of $f \in C_{0}\left(\mathbb{R}^{d}\right)$ by weighted polynomials $w^{-2 n} p_{2 n}$ uniformly on $\mathbb{R}^{d}$ is closely related to approximation by multivariate homogeneous polynomials

$$
h \in H_{n}^{d}:=\left\{\sum_{|\mathbf{k}|=n} a_{\mathbf{k}} \mathbf{x}^{\mathbf{k}}: a_{\mathbf{k}} \in \mathbb{R}\right\}
$$

of degree $n$ on boundaries of 0 -symmetric convex bodies. 
In this respect the following conjecture has been widely investigated in the past decade (see, for instance [2], [8], $[3],[7])$.

Conjecture 1. For any 0 -symmetric convex body $K \subset \mathbb{R}^{d}$ and every even $f \in C(\partial K)$ there exist homogeneous polynomials $h_{2 n} \in H_{2 n}^{d}$ such that $f=\lim _{n \rightarrow \infty} h_{2 n}$ uniformly on $\partial K$.

It is easy to see that the above conjecture is equivalent to the claim that every continuous function on $\partial K$ with $K$ as above can be uniformly approximated by the sum of 2 homogeneous polynomials, i.e., for every $f \in C(\partial K)$ we have that $f=\lim _{n \rightarrow \infty} h_{n}$ with certain $h_{n} \in H_{2 n}^{d}+H_{2 n+1}^{d}$ uniformly on $\partial K$.

This conjecture has been verified in the following three cases:

(i) when $d=2$ (see $[2,8])$;

(ii) for any 0 -symmetric convex polytope in $\mathbb{R}^{d}, d \geq 2$ (see [8]);

(iii) for any 0 -symmetric regular convex body in $\mathbb{R}^{d}, d \geq 2$ possessing a unique supporting hyperplane at every point on its boundary (see [3]).

Above results on homogeneous approximation will play an important role in our considerations below.

The outline of the paper is as follows. First a general result on density of weighted polynomials in $C_{0}\left(\mathbb{R}^{d}\right)$ in case of convex admissible weights will be given (Theorem 1). Then we proceed by exhibiting some model examples of convex weights for which density holds. Finally, Theorems 3 and 4 will deal with analogues questions for certain non convex weights. It will be shown that in non convex situation the density holds only for functions vanishing at a certain exceptional set related to the weight.

\section{New results}

Our first main result provides a Weierstrass type result asserting that uniform approximation on $\mathbb{R}^{d}$ by weighted polynomials $w^{-2 n} p_{2 n}$ is possible when the admissible weight is convex and, if $d>1$ is also piecewise $C^{1}$. The piecewise $C^{1}$ property of the weight $w$ is meant in the sence that $w$ is a maximum of finite number of $C^{1}$ weights.

Theorem 1 Let $w$ be a convex admissible weight on $\mathbb{R}^{d}, d \geq 1$. In addition, if $d>1$ assume that $w$ is piecewise $C^{1}$, i.e., with some $s \in \mathbb{N}$ we have $w=\max \left\{w_{j}: 1 \leq j \leq s\right\}$ where each $w_{j}$ is admissible convex and $w_{j}^{*} \in$ $C^{1}\left(\mathbb{R}^{d+1} \backslash\{0\}\right), 1 \leq j \leq s$. Then for every $f \in C_{0}\left(\mathbb{R}^{d}\right)$ there exist polynomials $p_{2 n} \in P_{2 n}^{d}$ so that

$$
w^{-2 n} p_{2 n} \rightarrow f, n \rightarrow \infty
$$

uniformly on $\mathbb{R}^{d}$.

Thus when $d=1$ the convexity of the admissible weight yields the density of weighted polynomials $w^{-2 n} p_{2 n}$ in the space $C_{0}(\mathbb{R})$. If $d>1$ we need in addition the piecewise $C^{1}$ smoothness of weights in order for the density to hold. It is plausible that the convexity of admissible weights suffices for the density of weighted polynomials $w^{-2 n} p_{2 n}$ in $C_{0}\left(\mathbb{R}^{d}\right), d>1$, as well. Thus we would like to offer the next conjecture which would provide a full analogue of weighted Weierstrass approximation theorem in $\mathbb{R}^{d}$.

Conjecture 2. For any convex admissible weight on $\mathbb{R}^{d}, d \geq 1$ and $f \in C_{0}\left(\mathbb{R}^{d}\right)$ there exist polynomials $p_{2 n} \in P_{2 n}^{d}$ so that

uniformly on $\mathbb{R}^{d}$.

$$
w^{-2 n} p_{2 n} \rightarrow f, n \rightarrow \infty
$$

Let us now give some model examples of weights for which the above theorem is applicable.

Example 1. For any $0<\alpha \leq \infty$ the $l_{\alpha}$ norm of $\mathbf{x}=\left(x_{1}, \ldots, x_{d}\right) \in \mathbb{R}^{d}$ is given by the relations

$$
|\mathbf{x}|_{\alpha}:=\left\{\begin{array}{l}
\left(\left|x_{1}\right|^{\alpha}+\ldots+\left|x_{d}\right|^{\alpha}\right)^{\frac{1}{\alpha}}, 0<\alpha<\infty \\
\max _{1 \leq j \leq d}\left|x_{j}\right|, \alpha=\infty .
\end{array}\right.
$$

Furthermore, for $\mathbf{x}=\left(x_{1}, \ldots, x_{d}\right) \in \mathbb{R}^{d}$ consider the weight

$$
w_{\alpha}(\mathbf{x}):=|(1, \mathbf{x})|_{\alpha}=\left\{\begin{array}{l}
\left(1+\left|x_{1}\right|^{\alpha}+\ldots+\left|x_{d}\right|^{\alpha}\right)^{\frac{1}{\alpha}}, 0<\alpha<\infty \\
\max \left\{1,\left|x_{1}\right|, \ldots,\left|x_{d}\right|\right\}, \alpha=\infty .
\end{array}\right.
$$

Now it easily follows that for this weight and $t>0$

$$
w_{\alpha}^{*}(\mathbf{x}, t)=t w_{\alpha}\left(\frac{\mathbf{x}}{t}\right)=|(t, \mathbf{x})|_{\alpha},(t, \mathbf{x}) \in \mathbb{R}^{d+1}
$$


is monotone increasing for every fixed $\mathbf{x} \in \mathbb{R}^{d}$. Moreover, clearly (1) holds with

$$
\tilde{w}_{\alpha}(\mathbf{x})=\lim _{t \rightarrow 0}|t| w_{\alpha}\left(\frac{\mathbf{x}}{t}\right)=|\mathbf{x}|_{\alpha}, \quad \mathbf{x} \in \mathbb{R}^{d} .
$$

Hence $w_{\alpha}(\mathbf{x})$ is an admissible weight for every $0<\alpha \leq \infty$. Furthermore, $w_{\alpha}(\mathbf{x})$ is also convex whenever $1 \leq \alpha \leq \infty$. In addition, when $1<\alpha<\infty$ these weights are $C^{1}$, i.e., $w_{\alpha}^{*} \in C^{1}\left(\mathbb{R}^{d+1} \backslash\{0\}\right)$ while for $\alpha=1$, $\infty$ the weights are piece wise $C^{1}$. Therefore for every $1 \leq \alpha \leq \infty$ weights $w_{\alpha}(\mathbf{x})$ defined in (3) provide a model of weights for which conditions of Theorem 1 hold. Hence we obtain the next

Corollary 2 Let $1 \leq \alpha \leq \infty, d \geq 1$. Then for every $f \in C_{0}\left(\mathbb{R}^{d}\right)$ there exist polynomials $p_{2 n} \in P_{2 n}^{d}$ so that

$$
w_{\alpha}^{-2 n} p_{2 n} \rightarrow f, n \rightarrow \infty
$$

uniformly on $\mathbb{R}^{d}$.

Example 2. It should be noted that the sufficient conditions imposed on the weight in Theorem 1 allow performing certain operations with the weights. In particular, the summation of the weights, or taking maximum of weights preserves the required properties. Therefore based on the weights (3) it follows for instance that Theorem 1 will also hold for weights

$$
w(\mathbf{x}):=\sum_{1 \leq j \leq d}\left(1+\left|x_{j}\right|^{\alpha_{j}}\right)^{\frac{1}{\alpha_{j}}}
$$

and

$$
w(\mathbf{x}):=\max _{1 \leq j \leq d}\left(1+\left|x_{j}\right|^{\alpha_{j}}\right)^{\frac{1}{\alpha_{j}}}
$$

whenever $1 \leq \alpha_{j}<\infty, 1 \leq j \leq d$. Note that in contrast to weights (3) the above weights are in general asymmetric with respect to the variables $x_{j}, 1 \leq j \leq d$.

Example 3. Another example of piece wise smooth weights for which Theorem 1 can be applied is provided by linear splines. Namely, we can take $w$ to be an even convex linear spline function on $\mathbb{R}^{d}$ in which case all requirements of Theorem 1 are fulfilled again.

While the admissibility of the weight appears to be a natural requirement for approximating every function in $C_{0}\left(\mathbb{R}^{d}\right)$ one may ask if the convexity of weight is also necessary for this approximation to hold, in general. A model example of non convex weights is given by weights (3) when $0<\alpha<1$. It turns out that convexity of the weight is crucial in order for the weighted approximation to hold for every $f \in C_{0}\left(\mathbb{R}^{d}\right)$.

Indeed, in a recent paper [4] the authors showed that in case $d=1$ and $0<\alpha<1$ there exist univariate weighted polynomials $w_{\alpha}^{-2 n} p_{2 n}, p_{n} \in P_{2 n}^{1}, n \in \mathbb{N}$, converging to $f \in C(\mathbb{R})$ uniformly on $\mathbb{R}$ if and only if $f(0)=$ $f(\infty)=f(-\infty)=0$. Here $f(\infty), f(-\infty)$ stand for the corresponding limits at infinity. Thus in case of these non convex admissible weights some additional restrictions need to be imposed on the functions which admit a univariate weighted polynomial approximation. Namely the function must vanish at a certain exceptional set. It is natural to expect that this phenomena will be preserved in multivariate setting, as well. The most interesting question here consists in finding the proper multidimensional exceptional sets.

So let us consider now approximation by weighted polynomials $w_{\alpha}^{-2 n} p_{2 n}, p_{2 n} \in P_{2 n}^{d}$ when $0<\alpha<1$, that is the weight is not convex.

Denote by

$$
L_{j}^{d}:=\left\{\mathbf{x}=\left(x_{1}, \ldots, x_{d}\right) \in \mathbb{R}^{d}: x_{j}=0\right\}, 1 \leq j \leq d
$$

the coordinate planes in $\mathbb{R}^{d}$, and let

$$
L^{d}:=\cup_{1 \leq j \leq d} L_{j}^{d}=\left\{\mathbf{x}=\left(x_{1}, \ldots, x_{d}\right) \in K_{\alpha}^{d}: x_{1} \cdot \ldots \cdot x_{d}=0\right\}
$$

be the union of all coordinate planes. Furthermore, we will write $f(\infty)=0$ if $f(\mathbf{y}) \rightarrow 0$ whenever $|\mathbf{y}| \rightarrow \infty$.

The next theorem is a multivariate extension of the result given in [4]. It identifies the exceptional zero set for the functions which admit weighted polynomial approximation on $\mathbb{R}^{d}$ with the non convex weights $w_{\alpha}, 0<\alpha<1$. Essentially our result shows that in multivariate case the exceptional zero set consists of the union of all coordinate planes $L^{d}$ and the infinity. 
Theorem 3 Let $0<\alpha<1$ and $d \geq 2$. Then in order for $f \in C_{0}\left(\mathbb{R}^{d}\right)$ to be a uniform limit on $\mathbb{R}^{d}$ of weighted polynomials $w_{\alpha}^{-2 n} p_{2 n}, p_{2 n} \in P_{2 n}^{d}$ it is necessary that $f=0$ on $L^{d} \cup\{\infty\}$. Moreover, if $0<\alpha<1$ is rational then any $f \in C\left(\mathbb{R}^{d}\right)$ which vanishes on $L^{d} \cup\{\infty\}$ is a uniform limit on $\mathbb{R}^{d}$ of weighted polynomials $w_{\alpha}^{-2 n} p_{2 n}, p_{2 n} \in P_{2 n}^{d}$.

As mentioned above uniform approximation in $C_{0}\left(\mathbb{R}^{d}\right)$ by weighted polynomials $w^{-2 n} p_{2 n}$ is closely related to the approximation by multivariate homogeneous polynomials on the boundaries of 0 -symmetric star like domains. So we will include now a companion to Theorem 3 related to homogeneous polynomial approximation.

Consider the $L_{\alpha}$ sphere in $\mathbb{R}^{d}$ given by

$$
K_{\alpha}^{d}:=\left\{\mathbf{x}=\left(x_{1}, \ldots, x_{d}\right) \in \mathbb{R}^{d}:\left|x_{1}\right|^{\alpha}+\ldots+\left|x_{d}\right|^{\alpha}=1\right\}=\left\{|\mathbf{x}|_{\alpha}=1\right\}, \quad \alpha>0 .
$$

When $0<\alpha<1$ the set $K_{\alpha}^{d}$ is not convex so the problem of homogeneous polynomial approximation on this set is not covered by the results mentioned in the Introduction. In Kroó-Totik [4] it is shown that if $d=2$ and $0<\alpha<1$ then in order that even function $f(x, y) \in C\left(K_{\alpha}^{2}\right)$ be a uniform limit on $K_{\alpha}^{2}$ of homogeneous polynomials $h_{2 n} \in H_{2 n}^{2}$ it is necessary and sufficient that $f( \pm 1,0)=f(0, \pm 1)=0$, i.e., the function must vanish at all the vertices of $K_{\alpha}^{2}$. Evidently, when $d>2$ the set of all non smooth points of $K_{\alpha}^{d}$ is given by

$$
K_{\alpha}^{d} \cap L^{d}=\left\{\mathbf{x}=\left(x_{1}, \ldots, x_{d}\right) \in K_{\alpha}^{d}: x_{1} \cdot \ldots \cdot x_{d}=0\right\},
$$

where as above $L^{d}$ is the union of all coordinate planes in $\mathbb{R}^{d}$. It turns out that only functions that vanish at these non smooth points admit homogeneous polynomial approximation on $K_{\alpha}^{d}$. Thus when $d>2$ the exceptional zero set consisting of all non smooth points is essentially wider than the set of all vertices of $K_{\alpha}^{d}$.

Theorem 4 Let $d>2,0<\alpha<1$. Then in order for an even function $f \in C\left(K_{\alpha}^{d}\right)$ to be a uniform limit on $K_{\alpha}$ of homogeneous polynomials $h_{2 n} \in H_{2 n}^{d}$ it is necessary that $f=0$ on $K_{\alpha}^{d} \cap L^{d}$. Moreover, if $0<\alpha<1$ is rational then the condition $f(\mathbf{x})=0, \mathbf{x} \in K_{\alpha}^{d} \cap L^{d}$ is also sufficient for this homogeneous polynomial approximation to hold.

Remark. It should be noted that Theorems 3 and 4 provide matching necessary and sufficient conditions for multivariate weighted and homogeneous approximation for rational $0<\alpha<1$. In [4] analogous results are given in the univariate case for every $0<\alpha<1$. In fact two proofs of sufficiency in Theorem 3 are given in [4] when $d=1$ : one using potential theoretic methods for every $0<\alpha<1$ and another one based on a explicit construction of approximating polynomials which works for rational $0<\alpha<1$. When $d \geq 2$ the needed potential theoretic methods are not available anymore, but nevertheless it will be shown below that for rational $0<\alpha<1$ the explicit construction of multivariate weighted approximating polynomials can still be accomplished even if $d \geq 2$. It appears to be plausible that sufficiency in Theorems 3 and 4 remains valid for irrational $0<\alpha<1$, as well. This remains an interesting open problem.

The next two sections contain the proofs of Theorems 1,3 and 4 . We will need to verify several auxiliary lemmas some of which are of independent interest. In particular, a general duality between weighted and homogeneous polynomial approximation will be exhibited, see Lemmas 2 and 5 below.

\section{Proof of Theorem 1}

We will need first to verify some auxiliary lemmas. The first lemma asserts that $w^{*}$ is convex on $\mathbb{R}^{d+1}$ for every admissible convex weight $w$, i.e., the homogenization of weights preserves convexity.

Lemma 1 For any admissible convex weight $w \in C\left(\mathbb{R}^{d}\right)$ it follows that $w^{*}(\mathbf{x}, t)$ is also a convex continuous weight on $\mathbb{R}^{d+1}$.

Proof. Since the weight $w$ is even it follows that $w^{*}(\mathbf{x}, t)=|t| w\left(\frac{\mathbf{x}}{t}\right), \mathbf{x} \in \mathbb{R}^{d}$ is even in variable $t$. Therefore for every $(\mathbf{x}, a),(\mathbf{y}, b) \in \mathbb{R}^{d+1}$ with some reals $a, b \neq 0, a+b \neq 0$ we have by the convexity of $w$

$$
\begin{gathered}
w^{*}(\mathbf{x}, a)+w^{*}(\mathbf{y}, b)=|a| w\left(\frac{\mathbf{x}}{|a|}\right)+|b| w\left(\frac{\mathbf{y}}{|b|}\right)= \\
(|a|+|b|)\left(\frac{|a|}{|a|+|b|} w\left(\frac{\mathbf{x}}{|a|}\right)+\frac{|b|}{|a|+|b|} w\left(\frac{\mathbf{y}}{|b|}\right)\right) \geq(|a|+|b|) w\left(\frac{\mathbf{x}+\mathbf{y}}{|a|+|b|}\right) .
\end{gathered}
$$


Furthermore, recalling that $t w\left(\frac{\mathbf{x}}{t}\right)$ is a monotone increasing function of $t>0$ for any $\mathbf{x} \in \mathbb{R}^{d}$ yields

$$
w^{*}(\mathbf{x}, a)+w^{*}(\mathbf{y}, b) \geq(|a|+|b|) w\left(\frac{\mathbf{x}+\mathbf{y}}{|a|+|b|}\right) \geq|a+b| w\left(\frac{\mathbf{x}+\mathbf{y}}{|a+b|}\right)=w^{*}(\mathbf{x}+\mathbf{y}, a+b) .
$$

The above inequality was derived for any $(\mathbf{x}, a),(\mathbf{y}, b) \in \mathbb{R}^{d+1}$ with $a, b \neq 0, a+b \neq 0$. But since $w^{*}(\mathbf{x}, t)$ is continuous on $\mathbb{R}^{d+1}$ it follows that

$$
w^{*}(\mathbf{x}, a)+w^{*}(\mathbf{y}, b) \geq w^{*}(\mathbf{x}+\mathbf{y}, a+b), \quad \forall(\mathbf{x}, a),(\mathbf{y}, b) \in \mathbb{R}^{d+1} .
$$

In addition, we evidently have $w^{*}(c \mathbf{x}, c t)=|c| w^{*}(\mathbf{x}, t), c \in \mathbb{R}$ which together with the above inequality verifies the convexity of $w^{*}(\mathbf{x}, t)$.

Our next lemma provides an important duality between weighted polynomial approximation on $\mathbb{R}^{d}$ and homogeneous polynomial approximation on star like domains in $\mathbb{R}^{d+1}$ associated with the corresponding weight.

Let $K \subset \mathbb{R}^{d}$ be a compact 0 -symmetric set with nonempty interior which is starlike with respect to the origin, that is for every $\mathbf{x} \in K$ we have that $(-\mathbf{x}, \mathbf{x}) \subset \operatorname{Int} K$.

When $K$ is a 0 -symmetric star like domain in $\mathbb{R}^{d}$ its Minkowski functional is defined by the relation

$$
\phi_{K}(\mathbf{x}):=\inf \left\{\alpha>0: \frac{\mathbf{x}}{\alpha} \in K\right\}, \mathbf{x} \in \mathbb{R}^{d} .
$$

Note that $\phi_{K}(\alpha \mathbf{x}):=|\alpha| \phi_{K}(\mathbf{x}), \alpha \in \mathbb{R}, \mathbf{x} \in \mathbb{R}^{d}$ and

$$
K=\left\{\mathbf{x} \in \mathbb{R}^{d}: \phi_{K}(\mathbf{x}) \leq 1\right\}, \partial K:=\left\{\mathbf{x} \in \mathbb{R}^{d}: \phi_{K}(\mathbf{x})=1\right\} .
$$

Let $w \in C\left(\mathbb{R}^{d}\right), d \geq 1$ be an admissible weight on $\mathbb{R}^{d}$. With this weight we associate a 0 -symmetric star like domain defined by

$$
K_{w}:=\left\{(\mathbf{x}, t) \in \mathbb{R}^{d+1}: w^{*}(\mathbf{x}, t) \leq 1\right\},
$$

where $w^{*}(\mathbf{x}, t)$ is given by the relation (2). Since $w^{*}(\mathbf{x}, t)$ is even, both in $\mathbf{x} \in \mathbb{R}^{d}$ and $t \in \mathbb{R}$, it follows that $K_{w}$ is symmetric with respect to $\mathbf{x} \in \mathbb{R}^{d}$ and $t \in \mathbb{R}$. Thus $K_{w}$ is a 0 -symmetric star like domain in $\mathbb{R}^{d+1}$. In addition it follows from Lemma 1 that $K_{w}$ is convex whenever $w$ is convex. Moreover, it is also easy to see that $w^{*}(\mathbf{x}, t)$ is the Minkowski functional of $K_{w}$.

Conversely, assume that $K$ is a 0 -symmetric star like set of points $(\mathbf{x}, t) \in \mathbb{R}^{d+1}$ which is also symmetric with respect to $\mathbf{x} \in \mathbb{R}^{d}$ for every fixed $t \in \mathbb{R}$, i.e., $(\mathbf{x}, t) \in K \Leftrightarrow(-\mathbf{x}, t) \in K, \forall t$. Then it is easy to see that its Minkowski functional $\phi_{K}(\mathbf{x}, t)$ is even both in $\mathbf{x} \in \mathbb{R}^{d}$ and $t \in \mathbb{R}$. Now we associate this set $K$ with an even positive weight on $\mathbb{R}^{d}$ defined by the relation

$$
w_{K}(\mathbf{x}):=\phi_{K}(\mathbf{x}, 1), \quad \mathbf{x} \in \mathbb{R}^{d} .
$$

Lemma 2 (i) Let $w \in C\left(\mathbb{R}^{d}\right), d \geq 1$ be an admissible weight on $\mathbb{R}^{d}$. Assume that for each $g \in C_{0}\left(\mathbb{R}^{d}\right)$ there exist polynomials $p_{2 n} \in P_{2 n}^{d}$ so that $w^{-2 n} p_{2 n} \rightarrow g, n \rightarrow \infty$ uniformly on $\mathbb{R}^{d}$. Then for every even function $f \in C\left(\partial K_{w}\right)$ there exist homogeneous polynomials $h_{2 n} \in H_{2 n}^{d+1}$ for which $f=\lim h_{2 n}$ uniformly on $\partial K_{w}$.

(ii) Conversely, let $K$ be any 0-symmetric star like set of points $(\mathbf{x}, t) \in \mathbb{R}^{d+1}$ which is symmetric with respect to $\mathbf{x} \in \mathbb{R}^{d}$ for every fixed $t \in \mathbb{R}$. Assume that for each even function $f \in C(\partial K)$ there exist homogeneous polynomials $h_{2 n} \in H_{2 n}^{d+1}$ such that $f=\lim h_{2 n}$ uniformly on $\partial K$. Then for every $g \in C_{0}\left(\mathbb{R}^{d}\right)$ there exist polynomials $p_{2 n} \in P_{2 n}^{d}$ so that $w_{K}^{-2 n} p_{2 n} \rightarrow g, n \rightarrow \infty$ uniformly on $\mathbb{R}^{d}$.

Proof. (i) By definition (2) it follows that for every $\mathbf{x} \in \mathbb{R}^{d}$

$$
w^{*}\left(\frac{\mathbf{x}}{w(\mathbf{x})}, \frac{1}{w(\mathbf{x})}\right)=1
$$

Since

this means that

$$
\partial K_{w}:=\left\{(\mathbf{x}, t) \in \mathbb{R}^{d+1}: w^{*}(\mathbf{x}, t)=1\right\}
$$

$$
\left(\frac{\mathbf{x}}{w(\mathbf{x})}, \frac{1}{w(\mathbf{x})}\right) \in \partial K_{w}, \quad \mathbf{x} \in \mathbb{R}^{d}
$$


Now given any even function $f \in C\left(\partial K_{w}\right)$ set

$$
g(\mathbf{x}):=f\left(\frac{\mathbf{x}}{w(\mathbf{x})}, \frac{1}{w(\mathbf{x})}\right), \quad \mathbf{x} \in \mathbb{R}^{d} .
$$

Since $f \in C\left(\partial K_{w}\right)$ and $w$ is positive and continuous this implies $g \in C\left(\mathbb{R}^{d}\right)$. Moreover, let us show that $g \in C_{0}\left(\mathbb{R}^{d}\right)$. Indeed using that $f$ is even it follows by (1) that as $|t| \rightarrow \infty$

$$
g(t \mathbf{x})=f\left(\frac{t \mathbf{x}}{w(t \mathbf{x})}, \frac{1}{w(t \mathbf{x})}\right) \rightarrow f\left(\frac{\mathbf{x}}{\tilde{w}(\mathbf{x})}, 0\right) \text { uniformly for } \mathbf{x} \in S^{d-1} .
$$

Thus $g \in C_{0}\left(\mathbb{R}^{d}\right)$ and hence there exist polynomials $p_{2 n} \in P_{2 n}^{d}$ so that $w^{-2 n} p_{2 n} \rightarrow g, n \rightarrow \infty$ uniformly on $\mathbb{R}^{d}$. Now set

$$
h_{2 n}(\mathbf{x}, t):=t^{2 n} p_{2 n}\left(\frac{\mathbf{x}}{t}\right) \in H_{2 n}^{d+1}
$$

and

$$
\partial K_{w}^{+}:=\left\{\mathbf{x}=\left(x_{1}, \ldots, x_{d+1}\right) \in K_{w}, x_{d+1}>0\right\} .
$$

Note that for any $(\mathbf{x}, t) \in \partial K_{w}^{+}$, we have $t w(\mathbf{x} / t)=1, t>0$. Thus using substitution $\mathbf{y}=\mathbf{x} / t \in \mathbb{R}^{d}$ and relation $t=1 / w(\mathbf{y})$ we arrive at

$$
f(\mathbf{x}, t)-h_{2 n}(\mathbf{x}, t)=f(\mathbf{x}, t)-t^{2 n} p_{2 n}\left(\frac{\mathbf{x}}{t}\right)=f\left(\frac{\mathbf{y}}{w(\mathbf{y})}, \frac{1}{w(\mathbf{y})}\right)-w^{-2 n}(\mathbf{y}) p_{2 n}(\mathbf{y})=g(\mathbf{y})-w^{-2 n}(\mathbf{y}) p_{2 n}(\mathbf{y}) .
$$

Since $w^{-2 n} p_{2 n} \rightarrow g, n \rightarrow \infty$ uniformly on $\mathbb{R}^{d}$ we obtain that $f=\lim h_{2 n}$ uniformly on $\partial K_{w}^{+}$. Since both $f$ and $h_{2 n}$ are even continuous functions the last statement clearly extends to all of $\partial K_{w} \square$.

(ii) For any $g \in C_{0}\left(\mathbb{R}^{d}\right)$ set

$$
f(\mathbf{x}, t):=\left\{\begin{array}{l}
g\left(\frac{\mathbf{x}}{t}\right), \mathbf{x} \in \mathbb{R}^{d}, t \in \mathbb{R} \backslash\{0\} \\
r_{g}(\mathbf{x}), t=0
\end{array}\right.
$$

where by the definition of the space $C_{0}\left(\mathbb{R}^{d}\right)$

$$
\lim _{|t| \rightarrow \infty} g(t \mathbf{x})=r_{g}(\mathbf{x}) \text { uniformly for } \mathbf{x} \in S^{d-1} .
$$

This easily yields that $f$ is continuous on $\mathbb{R}^{d+1} \backslash\{0\}$. In addition, it is clear that $f$ is even. Then by the assumption (ii) there exist homogeneous polynomials $h_{2 n} \in H_{2 n}^{d+1}$ such that $f=\lim _{n \rightarrow \infty} h_{2 n}$ uniformly on $\partial K$. Recall that for $(\mathbf{x}, t) \in \partial K \subset \mathbb{R}^{d+1}$ we have by the definition of $w_{K}$

$$
1=\phi_{K}(\mathbf{x}, t)=|t| \phi_{K}\left(\frac{\mathbf{x}}{t}, 1\right)=|t| w_{K}\left(\frac{\mathbf{x}}{t}\right),
$$

i.e., $|t|=w_{K}^{-1}\left(\frac{\mathbf{x}}{t}\right)$. Now we set $p_{2 n}(\mathbf{y}):=h_{2 n}(\mathbf{y}, 1) \in P_{2 n}^{d}$ and use again substitution $\mathbf{y}=\mathbf{x} / t \in \mathbb{R}^{d}$ yielding

$$
f(\mathbf{x}, t)-h_{2 n}(\mathbf{x}, t)=g\left(\frac{\mathbf{x}}{t}\right)-t^{2 n} h_{2 n}\left(\frac{\mathbf{x}}{t}, 1\right)=g(\mathbf{y})-w_{K}^{-2 n}(\mathbf{y}) p_{2 n}(\mathbf{y}), \quad \mathbf{y} \in \mathbb{R}^{d} .
$$

Since $f=\lim _{n \rightarrow \infty} h_{2 n}$ uniformly on $\partial K$ it follows that $w_{K}^{-2 n} p_{2 n} \rightarrow g, n \rightarrow \infty$ uniformly on $\mathbb{R}^{d}$. This completes the proof of the lemma.

The next lemma appears to be of independent interest. It shows that if weighted polynomial approximation on $\mathbb{R}^{d}$ holds for the admissible weights $w_{j}, 1 \leq j \leq s$ then it also holds for their maximum $w:=\max \left\{w_{j}, 1 \leq j \leq s\right\}$. The proof of this statement will be based on Lemma 2 and an elegant result of Varjú [8]. This latter states that given any two 0-symmetric star-like domains $K_{1}, K_{2} \in \mathbb{R}^{d}$ satisfying the homogeneous polynomial density Conjecture 1 formulated in the Introduction it follows that the same holds true for their intersection $K_{1} \cap K_{2}$, as well.

Lemma 3 Let $w_{j} \in C\left(\mathbb{R}^{d}\right), d \geq 1,1 \leq j \leq s$ be admissible weights on $\mathbb{R}^{d}$ such that each $g \in C_{0}\left(\mathbb{R}^{d}\right)$ is a uniform limit on $\mathbb{R}^{d}$ of some weighted polynomials $w_{j}^{-2 n} p_{2 n, j}, p_{2 n, j} \in P_{2 n}^{d}, 1 \leq j \leq s$ as $n \rightarrow \infty$. Then the same holds true with the weight $w:=\max \left\{w_{j}, 1 \leq j \leq s\right\}$. 
Proof. Assume that each $g \in C_{0}\left(\mathbb{R}^{d}\right)$ is a uniform limit on $\mathbb{R}^{d}$ of weighted polynomials $w_{j}^{-2 n} p_{2 n, j}, p_{2 n, j} \in$ $P_{2 n}^{d}, 1 \leq j \leq s$ as $n \rightarrow \infty$. Then by Lemma 2 (i) every even function $f \in C\left(\partial K_{w_{j}}\right)$ is a uniform limit on $\partial K_{w_{j}}$ of some homogeneous polynomials $h_{2 n, j} \in H_{2 n}^{d+1}, 1 \leq j \leq s$, where $K_{w_{j}}$ are the 0 -symmetric star like domains defined by $K_{w_{j}}:=\left\{(\mathbf{x}, t) \in \mathbb{R}^{d+1}: w_{j}^{*}(\mathbf{x}, t) \leq 1\right\}$, with $w_{j}^{*}(\mathbf{x}, t)$ given by relation (2) being the Minkowski functionals of $K_{w_{j}}$. Then by the result of Varjú [8] mentioned above the homogeneous polynomial density will hold for the 0-symmetric star like domain $K:=\cap_{1 \leq j \leq s} K_{w_{j}}$, as well. It is easy to see that the corresponding Minkowski functionals satisfy the relation

$$
\phi_{K}=\max \left\{\phi_{K_{j}}, 1 \leq j \leq s\right\}=\max \left\{w_{j}^{*}, 1 \leq j \leq s\right\}
$$

Furthermore, since for each even function $f \in C(\partial K)$ there exist homogeneous polynomials $h_{2 n} \in H_{2 n}^{d+1}$ such that $f=\lim _{n \rightarrow \infty} h_{2 n}$ uniformly on $\partial K$ it follows by Lemma 2 (ii) that for every $g \in C_{0}\left(\mathbb{R}^{d}\right)$ there exist polynomials $p_{2 n} \in P_{2 n}^{d}$ so that $w_{K}^{-2 n} p_{2 n} \rightarrow g, n \rightarrow \infty$ uniformly on $\mathbb{R}^{d}$, where by the previous relation and (2)

$$
w_{K}(\mathbf{x})=\phi_{K}(\mathbf{x}, 1)=\max \left\{w_{j}^{*}(\mathbf{x}, 1), 1 \leq j \leq s\right\}=\max \left\{w_{j}(\mathbf{x}), 1 \leq j \leq s\right\}, \quad \mathbf{x} \in \mathbb{R}^{d},
$$

i.e., weighted polynomial approximation on $\mathbb{R}^{d}$ also holds with the weight $w:=\max \left\{w_{j}, 1 \leq j \leq s\right\}$.

Now we are in position to verify Theorem 1. First let us note that based on Lemma 3 it suffices to prove the theorem for the case $s=1$. Thus consider a convex admissible weight $w$ on $\mathbb{R}^{d}, d \geq 1$ such that in addition, $w^{*}$ is $C^{1}$ on $\mathbb{R}^{d+1} \backslash\{0\}$ if $d>1$. Consider the 0 -symmetric star like domain associated with $w$ given by

$$
K_{w}:=\left\{(\mathbf{x}, t) \in \mathbb{R}^{d+1}: w^{*}(\mathbf{x}, t) \leq 1\right\} .
$$

Here as before $w^{*}(\mathbf{x}, t)$ given by relation (2) is the Minkowski functional of $K_{w}$ which is even in both $\mathbf{x} \in \mathbb{R}^{d}$ and $t \in \mathbb{R}$. Note that since $w$ is convex it follows by Lemma 1 that $w^{*}(\mathbf{x}, t)$ is convex too, and therefore $K_{w}$ is a 0 -symmetric convex domain in $\mathbb{R}^{d+1}$. Moreover, if $d>1$, i.e., $d+1>2$ then the Minkowski functionals of $K_{w}$ given by $w^{*}$ is $C^{1}$ which implies that $K_{w}$ is a regular 0 -symmetric convex domain in $\mathbb{R}^{d+1}$ when $d+1>2$. Then by the results on homogeneous polynomial approximation proved in [2],[8] and [3] for the case of convex bodies of dimension 2, and in case of any 0 -symmetric regular convex body, respectively (see the introduction above), it follows that for each even function $f \in C\left(\partial K_{w}\right)$ there exist homogeneous polynomials $h_{2 n} \in H_{2 n}^{d+1}$ such that $f=\lim _{n \rightarrow \infty} h_{2 n}$ uniformly on $\partial K_{w}$. Then by Lemma 2 (ii) for every $g \in C_{0}\left(\mathbb{R}^{d}\right)$ there exist polynomials $p_{2 n} \in P_{2 n}^{d}$ so that $w_{K_{w}}^{-2 n} p_{2 n} \rightarrow g, n \rightarrow \infty$ uniformly on $\mathbb{R}^{d}$. Here $w_{K_{w}}$ is the weight associated with $K_{w}$ which satisfies relations

$$
w_{K_{w}}(\mathbf{x}):=\phi_{K_{w}}(\mathbf{x}, 1)=w^{*}(\mathbf{x}, 1)=w(\mathbf{x}), \quad \mathbf{x} \in \mathbb{R}^{d} .
$$

Thus the required weighted polynomial approximation holds true for the weight $w$. This completes the proof of Theorem 1.

\section{Proof of Theorems 3 and 4}

Proof of necessity in Theorem 3. Assume now that $f \in C_{0}\left(\mathbb{R}^{d}\right)$ is a uniform limit on $\mathbb{R}^{d}$ of weighted polynomials $w_{\alpha}^{-2 n} p_{2 n}, p_{2 n} \in P_{2 n}^{d}$. In particular, this implies that for every $\mathbf{y} \in K_{\alpha}^{d},|\mathbf{y}|_{\alpha}=1$ the univariate function $g(t):=f(t \mathbf{y})$ is a uniform limit on $\mathbb{R}$ of univariate weighted polynomials $\left(1+|t|^{\alpha}\right)^{-2 n / \alpha} p_{2 n}, p_{2 n} \in P_{2 n}^{1}$. Then by [4], Theorem 1 $g(0)=g(\infty)=g(-\infty)=0$. Since $f \in C_{0}\left(\mathbb{R}^{d}\right)$ and thus $\lim _{|t| \rightarrow \infty} f(t \mathbf{x})=r_{f}(\mathbf{x})$ uniformly on $S^{d-1}$ it follows that $r_{f}(\mathbf{x})=0, \forall \mathbf{x} \in S^{d-1}$, i.e., $f(\infty)=0$. Furthermore, for any $1 \leq j \leq d$ and any $\mathbf{x}=\left(x_{1}, \ldots, x_{j-1}, 0, x_{j+1}, \ldots, x_{d}\right) \in L_{j}^{d}$ set $a:=\left(1+|\mathbf{x}|_{\alpha}^{\alpha}\right)^{1 / \alpha}$. Now consider the univariate function

$$
g(t):=f\left(x_{1}, \ldots, x_{j-1}, a t, x_{j+1}, \ldots, x_{d}\right) .
$$

Since $f=\lim _{n \rightarrow \infty} w_{\alpha}^{-2 n} p_{2 n}$ uniformly on $\mathbb{R}^{d}$ it obviously follows that

$$
g(t)=\lim _{n \rightarrow \infty}\left(1+|t|^{\alpha}\right)^{-2 n / \alpha} q_{2 n}, \quad q_{2 n}:=a^{-2 n} p_{2 n}\left(x_{1}, \ldots, x_{j-1}, a t, x_{j+1}, \ldots, x_{d}\right) \in P_{2 n}^{1} .
$$

Then using again [4], Theorem 1 we obtain $g(0)=f(\mathbf{x})=0$. Thus in addition to $f(\infty)=0$ we also have that $f=0$ on $L^{d}$.

Proof of necessity in Theorem 4. When $0<\alpha<1$ the $l_{\alpha}$ sphere in $\mathbb{R}^{d}$ given by

$$
K_{\alpha}^{d}=\left\{\mathbf{x} \in \mathbb{R}^{d}:|\mathbf{x}|_{\alpha}=1\right\}
$$


is not convex. In this case it has been verified in [4], Corollary 2 that if an even function $f(x, y) \in C\left(K_{\alpha}^{2}\right), d=$ $2,0<\alpha<1$ is a uniform limit on $K_{\alpha}^{2}$ of even homogeneous polynomials $f=\lim _{n \rightarrow \infty} h_{2 n}, h_{2 n} \in H_{2 n}^{d}$, then $f( \pm 1,0)=f(0, \pm 1)=0$, i.e., the function must vanish at all vertices of $K_{\alpha}^{2}$. Consider now arbitrary

$$
\left.\mathbf{y}=\left(y_{1}, \ldots y_{d}\right) \in K_{\alpha}^{d} \cap L^{d},|\mathbf{y}|_{\alpha}=1, y_{1} \cdot \ldots \cdot y_{d}=0\right\}, d \geq 2
$$

Then we can assume without loss of generality that $\mathbf{y}=\left(0, y_{2}, \ldots, y_{d}\right)$, and hence $\left|y_{2}\right|^{\alpha}+\ldots+\left|y_{d}\right|^{\alpha}=1$. Denote $\mathbf{a}_{1}:=(1,0, \ldots, 0) \in \mathbb{R}^{d}$ and let $M:=\operatorname{span}\left\{\mathbf{y}, \mathbf{a}_{1}\right\}$, be the 2 dimensional plane spanned by $\mathbf{y}, \mathbf{a}_{1}$. Then evidently

$$
\begin{gathered}
K_{\alpha}^{d} \cap M=\left\{t_{1} \mathbf{a}_{1}+t_{2} \mathbf{y} \in K_{\alpha}^{d}: t_{1}, t_{2} \in \mathbb{R}\right\}= \\
\left\{\left(t_{1}, t_{2}\right) \in \mathbb{R}^{2}:\left|t_{1}\right|^{\alpha}+\left|t_{2}\right|^{\alpha}\left(\left|y_{2}\right|^{\alpha}+\ldots+\left|y_{d}\right|^{\alpha}\right)=\left|t_{1}\right|^{\alpha}+\left|t_{2}\right|^{\alpha}=1\right\}
\end{gathered}
$$

is a 2 dimensional $l_{\alpha}$ sphere. Moreover, if the even function $f \in C\left(K_{\alpha}^{d}\right)$ is a uniform limit on $K_{\alpha}^{d}$ of homogeneous polynomials $h_{2 n} \in H_{2 n}^{d}$ then the bivariate continuous function $g\left(t_{1}, t_{2}\right):=f\left(t_{1} \mathbf{a}_{1}+t_{2} \mathbf{y}\right)$ is a uniform limit on $K_{\alpha}^{d} \cap M$ of corresponding bivariate homogeneous polynomials. Then using the bivariate result from [4] cited above it follows that $g( \pm 1,0)=g(0, \pm 1)=0$. Hence in particular, $g(0,1)=f(\mathbf{y})=0$. This verifies that any even function $f \in C\left(K_{\alpha}^{d}\right)$ which is a uniform limit on $K_{\alpha}^{d}$ of homogeneous polynomials $h_{2 n} \in H_{2 n}^{d}$ must vanish at every point of $K_{\alpha}^{d} \cap L^{d}$, i.e., the necessity in Theorem 4 follows.

Now we proceed to the more difficult task of verifying the sufficiency in Theorems 3 and 4.

First we present a lemma which establishes an equivalence between weighted polynomial approximation on $\mathbb{R}^{d}$ and homogeneous polynomial approximation on $K_{\alpha}^{d+1}$ in the presence of exceptional zero sets. This lemma and its proof is similar to the duality statement of Lemma 2 and therefore we only briefly outline its proof.

Lemma 5 Let $d \geq 1, \alpha>0$. Then the following statements are equivalent:

(i) For any $g \in C\left(\mathbb{R}^{d}\right)$ such that $g=0$ on $L^{d} \cup\{\infty\}$ there exist $p_{2 n} \in P_{2 n}^{d}$ so that

$$
g=\lim _{n \rightarrow \infty} w_{\alpha}^{-2 n}(\mathbf{y}) p_{2 n}(\mathbf{y})
$$

uniformly on $\mathbb{R}^{d}$.

(ii) Every even function $f \in C\left(K_{\alpha}^{d+1}\right)$ such that $f=0$ on $K_{\alpha}^{d+1} \cap L^{d+1}$ is a uniform limit on $K_{\alpha}^{d+1}$ of certain homogeneous polynomials $h_{2 n} \in H_{2 n}^{d+1}$ of degree $2 n$.

Proof. $\quad(i) \Rightarrow(i i)$ For any even function $f \in C\left(K_{\alpha}^{d+1}\right)$ such that $f=0$ on $K_{\alpha}^{d+1} \cap L^{d+1}$ set

$$
g(\mathbf{y}):=f\left(\frac{\mathbf{y}}{w_{\alpha}(\mathbf{y})}, \frac{1}{w_{\alpha}(\mathbf{y})}\right)=f(\mathbf{x}), \mathbf{y} \in \mathbb{R}^{d}, \mathbf{x} \in \mathbb{R}^{d+1} .
$$

As shown in the proof of Lemma 2 (i) it follows that $g \in C_{0}\left(\mathbb{R}^{d}\right)$. Now we need to show that in addition $g=0$ on $L^{d} \cup\{\infty\}$. First note that if $\mathbf{y}=\left(y_{1}, \ldots, y_{d}\right) \in L^{d}$, i.e., $y_{j}=0$ for some $1 \leq j \leq d$ then also $x_{j}=0$ yielding that $\mathbf{x} \in K_{\alpha}^{d+1} \cap L^{d+1}$. Hence $g(\mathbf{y})=f(\mathbf{x})=0$, i.e., $g=0$ on $L^{d}$. Furthermore, since

$$
w_{\alpha}(\mathbf{y}) \geq \max _{1 \leq j \leq d}\left|y_{j}\right| \geq d^{-\frac{1}{2}}|\mathbf{y}|
$$

it follows that $x_{d+1}=\frac{1}{w_{\alpha}(\mathbf{y})} \rightarrow 0$ whenever $|\mathbf{y}| \rightarrow \infty$. Thus using again that $f \in C\left(K_{\alpha}^{d+1}\right)$ and $f(\mathbf{x})=0$ if $x_{d+1}=0$ we obtain that $g(\mathbf{y})=f(\mathbf{x}) \rightarrow 0$ if $|\mathbf{y}| \rightarrow \infty$. Thus summarizing above observations we obtain that $g \in C_{0}\left(\mathbb{R}^{d}\right)$ and $g=0$ on $L^{d} \cup\{\infty\}$. Therefore by (i) with suitable $p_{2 n} \in P_{2 n}^{d}, n \in \mathbb{N}$ relation (6) must hold uniformly on $\mathbb{R}^{d}$. However, as shown in (4) for every $\mathbf{x} \in \partial K_{\alpha}^{d+1}, x_{d+1}>0$

$$
g(\mathbf{y})-w^{-2 n}(\mathbf{y}) p_{2 n}(\mathbf{y})=f(\mathbf{x})-h_{2 n}(\mathbf{x})
$$

where $h_{2 n}$ is a homogeneous polynomial of $\mathbf{x} \in \mathbb{R}^{d+1}$ of degree $2 n$. Thus since both $f$ and $h_{2 n}$ are even continuous functions it follows that $f=\lim h_{2 n}$ uniformly on $\partial K_{\alpha}^{d+1}$.

$($ ii $) \Rightarrow(i)$ Consider any $g \in C\left(\mathbb{R}^{d}\right), d \geq 1$ such that $g=0$ on $L^{d} \cup\{\infty\}$. Given $\mathbf{x} \in K_{\alpha}^{d+1}$ set

$$
f(\mathbf{x}):=\left\{\begin{array}{l}
g\left(\frac{x_{1}}{x_{d+1}}, \ldots, \frac{x_{d}}{x_{d+1}}\right), x_{d+1} \neq 0 \\
0, x_{d+1}=0
\end{array}\right.
$$


Evidently, since $g \in C_{0}\left(\mathbb{R}^{d}\right)$ and $g(\infty)=0$ it follows that $f \in C\left(K_{\alpha}^{d+1}\right)$. Furthermore, $f$ is even and since $g=0$ on $L^{d}$ we also have that $f=0$ on $K_{\alpha}^{d+1} \cap L^{d+1}$. Then by assumption (ii) $f=\lim h_{2 n}, h_{2 n} \in H_{2 n}^{d+1}$ uniformly on $K_{\alpha}^{d+1}$. Setting $y_{j}:=x_{j} / x_{d+1}, 1 \leq j \leq d$ for any $\mathbf{x}=\left(x_{1}, \ldots, x_{d+1}\right) \in K_{\alpha}^{d+1}, x_{d+1}>0$ we obtain

$$
x_{d+1}=\frac{1}{w_{\alpha}(\mathbf{y})}, x_{j}=\frac{y_{j}}{w_{\alpha}(\mathbf{y})}, 1 \leq j \leq d
$$

and hence as in (5)

uniformly on $\mathbb{R}^{d} \square$.

$$
f(\mathbf{x})-h_{2 n}(\mathbf{x})=g(\mathbf{y})-w_{\alpha}^{-2 n}(\mathbf{y}) p_{2 n}(\mathbf{y}) \rightarrow 0
$$

The next lemma provides an explicit construction of certain multivariate polynomials which are used for multivariate weighted approximation in $\mathbb{R}^{d}, d \geq 2$.

Lemma 6 Let $\alpha=\frac{p}{q}, p, q \in \mathbb{N}$ and $t_{k}:=e^{\frac{\pi k i}{q}}, 1 \leq k \leq 2 q$, be the $2 q$-th roots of unity. Then for any $n \in \mathbb{N}$ and $\mathbf{x} \in \mathbb{R}^{d}$

$$
Q_{n}(\mathbf{x}):=\left|x_{1}\right|^{\alpha} \ldots\left|x_{d}\right|^{\alpha} \sum_{1 \leq s_{j} \leq 2 q, 1 \leq j \leq d} t_{s_{1}} \ldots t_{s_{d}}\left(1+t_{s_{1}}\left|x_{1}\right|^{\alpha}+\ldots+t_{s_{d}}\left|x_{d}\right|^{\alpha}\right)^{n q} \in P_{p n+d}^{d}
$$

Proof. We use the relation

$$
\sum_{k=1}^{2 q} t_{k}^{l}=0
$$

which holds for any integer $l \neq 2 r q, r \in \mathbb{N}$. To verify that, it suffices to note that, by the periodicity of roots of unity, $\sum_{k=1}^{2 q} t_{k}^{l}=t_{1}^{l} \sum_{k=1}^{2 q} t_{k}^{l}$. Hence if $t_{1}^{l} \neq 1$, then the above relation must hold. In addition, we evidently have $\sum_{k=1}^{2 q} t_{k}^{l}=2 q$ whenever $l=2 r q, r \in \mathbb{N}$.

Using these relations and the multinomial formula we have for any $\mathbf{u}=\left(u_{1}, \ldots u_{d}\right) \in \mathbb{R}^{d}$

$$
\begin{aligned}
& Q(\mathbf{u}):=u_{1} \ldots u_{d} \sum_{1 \leq s_{j} \leq 2 q, 1 \leq j \leq d} t_{s_{1}} \ldots t_{s_{d}}\left(1+t_{s_{1}} u_{1}+\ldots+t_{s_{d}} u_{d}\right)^{n q} \\
& =u_{1} \ldots u_{d} \sum_{1 \leq s_{j} \leq 2 q, 1 \leq j \leq d} t_{s_{1}} \ldots t_{s_{d}} \sum_{m_{1}+\ldots+m_{d} \leq n q} b_{\mathbf{m}}\left(t_{s_{1}} u_{1}\right)^{m_{1}} \ldots\left(t_{s_{d}} u_{d}\right)^{m_{d}} \\
& \sum_{m_{1}+\ldots+m_{d} \leq n q} b_{\mathbf{m}} u_{1}^{m_{1}+1} \ldots u_{d}^{m_{d}+1} \prod_{1 \leq j \leq d} \sum_{1 \leq s_{j} \leq 2 q} t_{s_{j}}^{m_{j}+1}
\end{aligned}
$$

with proper multinomial coefficients $b_{\mathbf{m}}$. Clearly, we have for the product

$$
\prod_{1 \leq j \leq d} \sum_{1 \leq s_{j} \leq 2 q} t_{s_{j}}^{m_{j}+1}=(2 q)^{d}
$$

whenever $m_{j}+1=2 q r_{j}, 1 \leq j \leq d$ for some integers $r_{j} \in \mathbb{N}$, and otherwise this product is always zero. Thus we obtain that

$$
Q(\mathbf{u})=\sum_{2 r_{1}+\ldots+2 r_{d} \leq n+\frac{d}{q}} c_{\mathbf{r}} u_{1}^{2 q r_{1}} \ldots u_{d}^{2 q r_{d}}
$$

with some $c_{\mathbf{r}} \in \mathbb{R}$. Hence setting $u_{j}:=\left|x_{j}\right|^{\alpha}=|x|^{\frac{p}{q}}, 1 \leq j \leq d$ it follows that $u_{j}^{2 q r_{j}}=x_{j}^{2 p r_{j}}, 1 \leq j \leq d$ and therefore

$$
Q_{n}(\mathbf{x}):=Q\left(\left|x_{1}\right|^{\alpha}, \ldots,\left|x_{d}\right|^{\alpha}\right)=\sum_{2 r_{1}+\ldots+2 r_{d} \leq n+\frac{d}{q}} c_{\mathbf{r}} x_{1}^{2 p r_{1}} \ldots x_{d}^{2 p r_{d}} \in P_{p n+d}^{d} .
$$

Lemma 7 Let $0<\alpha=\frac{p}{q}, p, q \in \mathbb{N}$ and $Q_{n} \in P_{p n+d}^{d}$ be the polynomial given by (7). Then for any polynomial $G \in P_{p}^{d}$ of degree at most $p$ we have

$$
\left\|\frac{G(\mathbf{x}) Q_{n}(\mathbf{x})}{w_{\alpha}(\mathbf{x})^{p(n+d+2)}}-\frac{G(\mathbf{x})\left|x_{1} \ldots x_{d}\right|^{\alpha}}{w_{\alpha}(\mathbf{x})^{p(d+2)}}\right\|_{C\left(\mathbb{R}^{d}\right)}=O\left(\frac{1}{n}\right), n \in \mathbb{N} .
$$


Proof. We will need the following identity which holds for any given $a>0$ and $t_{k}=e^{\frac{\pi k i}{q}}, 1 \leq k \leq 2 q$

$$
\left|1+t_{k} a\right|^{2}=\left(1+a \Re t_{k}\right)^{2}+a^{2}\left(\Im t_{k}\right)^{2}=(1+a)^{2}+2 a \Re t_{k}-2 a=(1+a)^{2}-4 a \sin ^{2} \frac{\pi k}{2 q} .
$$

Evidently it implies that whenever $1 \leq k \leq 2 q-1$, i.e, $t_{k} \neq 1$

$$
\left|1+t_{k} a\right| \leq(1+a) \sqrt{1-\frac{4 a}{(1+a)^{2}} \sin ^{2} \frac{\pi k}{2 q}} \leq 1+a-\frac{2 a}{1+a} \sin ^{2} \frac{\pi}{2 q}=1+a-\frac{\gamma_{q} a}{1+a}, \quad \gamma_{q}:=2 \sin ^{2} \frac{\pi}{2 q} .
$$

Iterating the above estimate further consider the sum $\left|1+t_{s_{1}} a_{1}+\ldots+t_{s_{d}} a_{d}\right|, a_{j}>0,1 \leq s_{j} \leq 2 q$ assuming that $t_{s_{d}} \neq 1$. Then by $(9)$

$$
\left|1+t_{s_{1}} a_{1}+\ldots+t_{s_{d}} a_{d}\right| \leq a_{1}+\ldots+a_{d-1}+\left|1+t_{s_{d}} a_{d}\right| \leq 1+a_{1}+\ldots+a_{d}-\frac{\gamma_{q} a_{d}}{1+a_{d}} .
$$

Now we will apply relation (7) which yields using notation $|\mathbf{x}|_{\alpha}:=\left(\left|x_{1}\right|^{\alpha}+\ldots+\left|x_{d}\right|^{\alpha}\right)^{\frac{1}{\alpha}}$

$$
\frac{Q_{n}\left(x_{1}, \ldots, x_{d}\right)}{\left(1+|\mathbf{x}|_{\alpha}^{\alpha}\right)^{q n+s}}=\frac{\left|x_{1} \ldots x_{d}\right|^{\alpha}}{\left(1+|\mathbf{x}|_{\alpha}^{\alpha}\right)^{s}}+R\left(x_{1}, \ldots, x_{d}\right)
$$

where

$$
R\left(x_{1}, \ldots, x_{d}\right):=\frac{\left|x_{1} \ldots x_{d}\right|^{\alpha} \sum^{*} t_{s_{1}} \ldots t_{s_{d}}\left(1+t_{s_{1}}\left|x_{1}\right|^{\alpha}+\ldots+t_{s_{d}}\left|x_{d}\right|^{\alpha}\right)^{n q}}{\left(1+|\mathbf{x}|_{\alpha}^{\alpha}\right)^{q n+s}}
$$

with the symbol $\sum^{*}$ indicating summation over all $1 \leq s_{j} \leq 2 q, 1 \leq j \leq d$ except the term corresponding to $s_{1}=\ldots=s_{d}=2 q$. Thus in each term of $\sum^{*}$ at least one of $t_{j}$-s is distinct from 1 . Therefore using relation (10) and the symmetry we obtain that for each term in $\sum^{*}$

$$
\left\|\frac{\left|x_{1} \ldots x_{d}\right|^{\alpha}\left(1+t_{s_{1}}\left|x_{1}\right|^{\alpha}+\ldots+t_{s_{d}}\left|x_{d}\right|^{\alpha}\right)^{n q}}{\left(1+|\mathbf{x}|_{\alpha}^{\alpha}\right)^{q n+s}}\right\|_{C\left(\mathbb{R}^{d}\right)} \leq\left\|\frac{\left|x_{1} \ldots x_{d}\right|^{\alpha}\left(1+|\mathbf{x}|_{\alpha}^{\alpha}-\frac{\gamma_{q}\left|x_{d}\right|^{\alpha}}{1+\left|x_{d}\right|^{\alpha}}\right)^{n q}}{\left(1+|\mathbf{x}|_{\alpha}^{\alpha}\right)^{q n+s}}\right\|_{C\left(\mathbb{R}^{d}\right)}
$$

Thus for the remainder term in (11) we obtain the next estimate

$$
\|R\|_{C\left(\mathbb{R}^{d}\right)} \leq(2 q)^{d}\left\|\frac{\left|x_{1} \ldots x_{d}\right|^{\alpha}\left(1+|\mathbf{x}|_{\alpha}^{\alpha}-\frac{\gamma_{q}\left|x_{d}\right|^{\alpha}}{1+\left|x_{d}\right|^{\alpha}}\right)^{n q}}{\left(1+|\mathbf{x}|_{\alpha}^{\alpha}\right)^{q n+s}}\right\|_{C\left(\mathbb{R}^{d}\right)} .
$$

We will now give an upper bound for the norm of the above function using notations

$$
v(\mathbf{x})=v\left(x_{1}, \ldots, x_{d}\right):=1+|\mathbf{x}|_{\alpha}^{\alpha}, \quad u(\mathbf{x})=u\left(x_{1}, \ldots, x_{d}\right):=\frac{\left|x_{d}\right|^{\alpha}}{\left(1+\left|x_{d}\right|^{\alpha}\right) v(\mathbf{x})} .
$$

Applying these notations in the above estimate for $\|R\|_{C\left(\mathbb{R}^{d}\right)}$ and using that

$$
\left|x_{1} \ldots x_{d-1}\right|^{\alpha} \leq v(\mathbf{x})^{d-1}, \quad\left|x_{d}\right|^{\alpha} \leq v(\mathbf{x})^{2} u(\mathbf{x})
$$

clearly implies

$$
\|R\|_{\mathbb{R}^{d}} \leq(2 q)^{d}\left\|\left.\left|x_{1}\right| \ldots x_{d}\right|^{\alpha} \frac{\left(1-\gamma_{q} u(\mathbf{x})\right)^{n q}}{v(\mathbf{x})^{s}}\right\|_{C\left(\mathbb{R}^{d}\right)} \leq(2 q)^{d}\left\|\frac{u(\mathbf{x})\left(1-\gamma_{q} u(\mathbf{x})\right)^{n q}}{v(\mathbf{x})^{s-d-1}}\right\|_{C\left(\mathbb{R}^{d}\right)} .
$$

Now recalling that $s \geq d+1$ and using that $v(\mathbf{x}) \geq 1, \mathbf{x} \in \mathbb{R}^{d}$ we obtain

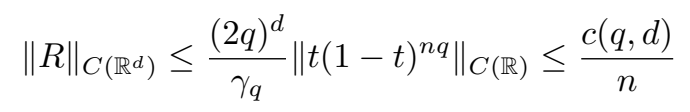


with a constant $c(q, d)$ depending only on $q$ and $d$. Applying this estimate in (11) yields

$$
\left|\frac{Q_{n}(\mathbf{x})}{\left(1+|\mathbf{x}|_{\alpha}^{\alpha}\right)^{q n+s}}-\frac{\left|x_{1} \ldots x_{d}\right|^{\alpha}}{\left(1+|\mathbf{x}|_{\alpha}^{\alpha}\right)^{s}}\right| \leq \frac{c(q, d)}{n}, \quad \mathbf{x}=\left(x_{1}, \ldots, x_{d}\right) \in \mathbb{R}^{d}, n \in \mathbb{N} .
$$

Now we can observe that for any polynomial $G \in P_{p}^{d}$ of degree at most $p$ we have

$$
\left\|\frac{G}{w_{\alpha}^{p}}\right\|_{C\left(\mathbb{R}^{d}\right)} \leq c
$$

Thus multiplying relation (12) by the bounded quantity $\left|\frac{G(\mathbf{x})}{w_{\alpha}(\mathbf{x})^{p}}\right|$ and setting $s:=\frac{p(d+1)}{\alpha}>d+1$ in (12) yields

$$
\left\|\frac{G(\mathbf{x}) Q_{n}(\mathbf{x})}{w_{\alpha}(\mathbf{x})^{p(n+d+2)}}-\frac{G(\mathbf{x})\left|x_{1} \ldots x_{d}\right|^{\alpha}}{w_{\alpha}(\mathbf{x})^{p(d+2)}}\right\|_{C\left(\mathbb{R}^{d}\right)}=O\left(\frac{1}{n}\right)
$$

which is the statement of the lemma.

Proof of sufficiency in Theorems 3 and 4. Now the proof of the sufficiency in Theorem 3 can be finalized by using a Stone-Weierstrass-type argument. For any closed subalgebra of functions $A \subset C(K)$ with $K$ being a compact Hausdorff space denote by $Z_{A}:=\{x \in K: g(x)=0$, for all $g \in A\}$ the zero set of $A$. Then the Stone-Weierstrass theorem (see e.g., [1, p. 13]) states that if $A$ separates points in $K \backslash Z_{A}$, then any $f \in C(K)$ which vanishes on $Z_{A}$ belongs to $A$, i.e., $A=\left\{f \in C(K): f=0\right.$ on $\left.Z_{A}\right\}$. We will use this statement in the space

$$
C^{*}\left(\mathbb{R}^{d}\right):=\left\{f \in C\left(\mathbb{R}^{d}\right): f(\infty)\right\}=0
$$

of continuous functions vanishing at infinity which clearly provides the needed compactification of the domain.

Let $\alpha=\frac{p}{q}, p, q \in \mathbb{N}, 0<\alpha<1$. The proof will be accomplished in two steps. First we will verify the need statement for the subsequence $\mathbb{N}_{p}:=\{p n: n \in \mathbb{N}\}$.

Set

$$
A_{p}:=\left\{f \in C^{*}\left(\mathbb{R}^{d}\right): f=\lim _{n \rightarrow \infty} w_{\alpha}^{-p n} g_{p n}, g_{p n} \in P_{p n}^{d}\right\} .
$$

Obviously, $A_{p}$ is a closed subalgebra of $C^{*}\left(\mathbb{R}^{d}\right)$, where by the already proven necessary condition in Theorem 3 we have $L^{d} \cup\{\infty\} \in Z_{A_{p}}$. Furthermore it follows by Lemma 7 that for any polynomial $G \in P_{p}^{d}$ of degree at most $p$ we have

$$
G(\mathbf{x})\left|x_{1} \ldots x_{d}\right|^{\alpha} w_{\alpha}(\mathbf{x})^{-p(d+2)} \in A_{p} .
$$

Since function $\left|x_{1} \ldots x_{d}\right|^{\alpha} w_{\alpha}(\mathbf{x})^{-p(d+2)}$ vanishes only on $L^{d} \cup\{\infty\}$ it follows that $L^{d} \cup\{\infty\}=Z_{A_{p}}$. Moreover polynomials $G \in P_{p}^{d}, p \geq 1$ separate points in all of $\mathbb{R}^{d}$ and hence functions (13) separate points in $\mathbb{R}^{d} \backslash\left\{L^{d} \cup\{\infty\}\right\}$. Thus any function $f \in C\left(\mathbb{R}^{d}\right)$ which vanishes on $L^{d} \cup\{\infty\}$ is in $A_{p}$ i.e., is a uniform limit on $\mathbb{R}^{d}$ of weighted polynomials $w_{\alpha}^{-p n} g_{p n}, g_{p n} \in P_{p n}^{d}$.

Now for any $0 \leq j \leq p-1$ and any polynomial $G \in P_{1}^{d}$ of degree at most 1 consider continuous functions

$$
f_{j}(\mathbf{x}):=x_{1} \ldots x_{d} G(\mathbf{x}) w_{\alpha}(\mathbf{x})^{-p-d+j-1}, \quad 0 \leq j \leq p-1 .
$$

It can be easily verified that $f_{j}$ vanishes on $L^{d} \cup\{\infty\}=Z_{A_{p}}$ for every $0 \leq j \leq p-1$. Thus by the previous step of the proof each $f_{j}$ is a uniform limit on $\mathbb{R}^{d}$ of weighted polynomials $w_{\alpha}^{-p n} g_{j, n}, g_{j, n} \in P_{p n}^{d}, 0 \leq j \leq p-1$. Of course this is equivalent to

$$
w_{\alpha}(\mathbf{x})^{-p n-j} g_{j, n}(\mathbf{x}) \rightarrow x_{1} \ldots x_{d} G(\mathbf{x}) w_{\alpha}(\mathbf{x})^{-p-d-1} \in C^{*}\left(\mathbb{R}^{d}\right), \quad 0 \leq j \leq p-1, \quad n \rightarrow \infty .
$$

Now for an $m \in \mathbb{N}, m \equiv j(\bmod p)$, say for $m=p n+j$ with some $n \in \mathbb{N}$ and $0 \leq j \leq p-1$, set

$$
g_{m}:=g_{j, n} \in P_{p n}^{d} \subset P_{m}^{d} .
$$

Then the last relation means that we found a sequence of polynomials $g_{m} \in P_{m}^{d}, m=1,2, \ldots$, such that

$$
w_{\alpha}(\mathbf{x})^{-m} g_{m}(\mathbf{x}) \rightarrow x_{1} \ldots x_{d} G(\mathbf{x}) w_{\alpha}(\mathbf{x})^{-p-d-1} \in C^{*}\left(\mathbb{R}^{d}\right), \quad 0 \leq j \leq p-1, \quad n \rightarrow \infty .
$$


Therefore, if we define

$$
A:=\left\{f \in C^{*}\left(\mathbb{R}^{d}\right): f=\lim _{m \rightarrow \infty} w_{\alpha}^{-m} g_{m}, g_{m} \in P_{m}^{d}\right\}
$$

then it follows that $x_{1} \ldots x_{d} G(\mathbf{x}) w_{\alpha}(\mathbf{x})^{-p-d-1} \in A$ for any polynomial $G \in P_{1}^{d}$ of degree at most 1 . Just as above this means that $Z_{A}=L^{d} \cup\{\infty\}$ and we have suitable test functions in the closed subalgebra $A \subset C^{*}\left(\mathbb{R}^{d}\right)$ which separate points in $\mathbb{R}^{d} \backslash\left\{L^{d} \cup\{\infty\}\right\}$. Hence, repeated application of the Stone-Weierstrass theorem implies that any function $f \in C\left(\mathbb{R}^{d}\right)$ which vanishes on $L^{d} \cup\{\infty\}$ is in $A$ i.e., is a uniform limit on $\mathbb{R}^{d}$ of weighted polynomials $w_{\alpha}^{-n} g_{n}, \quad g_{n} \in P_{n}^{d}$. This completes the proof of the sufficiency in Theorem 3. Moreover, recalling Lemma 5 we obtain the sufficiency in Theorem 4, as well.

Conclusions. As it can be seen from the above results convexity of the weight plays a crucial role in the study of density of weighted polynomial approximation. In this respect it would be very important to verify that density holds for every convex admissible weight without the assumption of piecewise $C^{1}$ smoothness as stated in Conjecture 2 for $d>1$. Another promising line of investigation could be related to the study of non convex weights. As it is indicated by Theorem 3 in non convex case certain exceptional zero sets come into play and finding these sets poses a rather nontrivial question. Furthermore, the study of weighted polynomial approximation in $C_{0}\left(\mathbb{R}^{d}\right)$ might lead to a further insight into the question of density of multivariate homogeneous polynomials.

\title{
References
}

[1] R. DeVore, G.G. Lorentz, Constructive approximation, Grundlehren der mathematischen Wissenschaften, 303, Springer Verlag, 1993.

[2] D. Benko and A. Kroó, A Weierstrass-type theorem for homogeneous polynomials, Trans. Amer. Math. Soc., 361(2009), 1645-1665.

[3] A. Kroó and J. Szabados, On the density of homogeneous polynomials on regular convex surfaces, Acta Sci. Math., 75(2009), 143-159.

[4] A. Kroó and V. Totik, Density of bivariate homogeneous polynomials on non-convex curves, Proc. Amer. Math. Soc., 147(2019), 167-177.

[5] E. Saff and V. Totik, Logarithmic potentials with external fields, Grundlehren der mathematischen Wissenschaften, 316, Springer Verlag, Berlin, 1997.

[6] V. Totik, Weighted approximation with varying weights, Lecture Notes in Mathematics, 1569, Springer-Verlag, Berlin-Heidelberg, 1994.

[7] V. Totik, Approximation by homogeneous polynomials, J. Approx. Th., 174 (2013), $192-205$.

[8] P. Varjú, Approximation by homogeneous polynomials, Constr. Approx., 26,(2007), 317-337.

\author{
András Kroó \\ Alfréd Rényi Institute of Mathematics \\ Hungarian Academy of Sciences \\ Budapest, Hungary \\ and \\ Budapest University of Technology and Economics \\ Department of Analysis, Budapest, Hungary
}

